University of Nebraska - Lincoln

DigitalCommons@University of Nebraska - Lincoln

April 1987

\title{
Radiosensitivity Parameters for Neoplastic Transformations in C3H10T1/2 Cells
}

\author{
M. P. R. Waligorski \\ Institute of Nuclear Physics, Krakow, Poland \\ G. L. Sinclair \\ University of Nebraska-Lincoln \\ Robert Katz \\ University of Nebraska-Lincoln, rkatz2@unl.edu
}

Follow this and additional works at: https://digitalcommons.unl.edu/physicskatz

Part of the Physics Commons

Waligorski, M. P. R.; Sinclair, G. L.; and Katz, Robert, "Radiosensitivity Parameters for Neoplastic Transformations in C3H10T1/2 Cells" (1987). Robert Katz Publications. 32.

https://digitalcommons.unl.edu/physicskatz/32

This Article is brought to you for free and open access by the Research Papers in Physics and Astronomy at DigitalCommons@University of Nebraska - Lincoln. It has been accepted for inclusion in Robert Katz Publications by an authorized administrator of DigitalCommons@University of Nebraska - Lincoln. 


\title{
Radiosensitivity Parameters for Neoplastic Transformations in C3H10T1/2 Cells
}

\author{
M. P. R. Waligorski, * G. L. Sinclair, and Robert Katz \\ University of Nebraska-Lincoln, Lincoln, Nebraska 68588-0111 \\ * IAEA Fellow. Permanent address: Institute of Nuclear Physics, Krakow, Poland
}

\begin{abstract}
We have evaluated radiosensitivity parameters for cellular transformation from published experimental data on neoplastic transformations induced in C3H10T1/2 cells by BEVALAC ions. The measured RBE values are well reproduced by a track theory calculation using sets of $m$-target parameters with either $m=2$ or $m=3$, suggesting a quadratic or cubic extrapolation to low doses of $\gamma$ rays. Using track theory one is thus able to predict transformation frequencies in those cells after an arbitrary radiation field, under known or assumed conditions of exposure, in a manner shown earlier for cellular survival. Extension of these calculations to interpret cancer incidence in vivo is also discussed.
\end{abstract}

\section{Introduction}

We are as yet unable to follow the chain of events which lead from radiation-induced damage at the molecular level through effects such as neoplastic transformation apparent at the cellular level to the expression of radiation-induced cancer in man. When seeking dose-effect relationships in the epidemiology of radiation-induced cancer and in radiation-induced neoplastic cell transformation, we would like to assume that in vivo and in vitro effects of radiation can be interrelated in a manner simple enough to be able to project, e.g., cancer incidence, from observations made at the cellular level. A radiobiological model invoked to address the dose-effect relationship at the cellular level should be capable of systematizing the available experimental data and of predicting the outcome of experiments it purports to classify, over a wide range of doses and radiation qualities. Of special importance is the ability of the model to predict correctly the relative biological effectiveness (RBE) of different radiations for specified end points in various biological systems. The track structure theory of RBE (1-3) appears to satisfy these postulates with respect to cellular survival. The theory requires the knowledge of four radiosensitivity parameters, two of which ( $m$, the number of targets per cell, and $E_{0}$, the characteristic X-ray dose) are extracted in principle from the response of the system to $\mathrm{X}$ or ${ }^{60} \mathrm{Co}$ irradiation. The remaining two $\left(\sigma_{0}\right.$, loosely interpreted as the cross-sectional area of the "cell nucleus," within which the inactivation sites are presumably located, and $\kappa$, 
a measure of the size of the inactivation site itself) are found in principle from survival measurements after track segment irradiations by energetic charged particles. In practice, all four parameters are fitted simultaneously to the entire set of data. The theory states that the parameters of the cellular system (as specified by these four numbers) and the parameters of the beam irradiation (as given by the effective charge value $Z^{*}$ and the relative velocity $\beta$ of the beam particles) are not separable variables. One consequence, confirmed experimentally $(4,5)$ is that RBE is not a single-valued function of any irradiation parameter, such as LET or $Z^{* 2} / \beta^{2}$. Another is the experimentally observed displacement with $Z$ and the decline in cellular action cross sections with an increase in ion LET for mammalian cells irradiated with UNILAC ions, interpreted here as a track "thindown" effect (6).

Neoplastic cell transformations in the C3H10T1/2 mammalian cell line after irradiation with beams of several different energetic heavy ions accelerated in the BEVALAC facility have been studied for several years by Yang and colleagues, and the collected experimental results have been published recently (7). Using these results we were able to establish the radiosensitivity parameters and to test the applicability of the model for predicting oncogenic radiation-induced transformations in this cell system. We then proceed, with caution, to a discussion of the possibility of applying our calculations to interpret cancer incidence in vivo in the Harderian gland of pituitary-isografted mice exposed to heavy ions (8). Our focus is on the dose dependence of transformation frequency as extrapolated to low X-ray doses. This analysis reinforces our belief that the issue of the initial slope for low doses of low-LET radiations should be addressed through the response of biological systems to high doses of high-LET radiations, as interpreted by a theory of RBE which accounts for the major contribution of energetic $\delta$ rays, recently shown to dominate the response at very high values of LET.

\section{The Model}

Detailed descriptions of the cellular track model have been given elsewhere (1-3). Here we present only the main concepts of the model and list the equations used in our calculations. Following our earlier studies of the appearance of particle tracks in nuclear emulsion (9), we distinguish between the "grain-count" regime, where inactivations occur randomly along the particle's path, and the "track-width" regime, where the inactivations are distributed like a "hairy rope." The transition from the grain-count to trackwidth regime takes place in the neighborhood of $Z^{* 2} / \kappa \beta^{2}$ of about 4 ; at lower values we are in the grain-count regime, and at higher values in the track-width regime. As we assume that cells are able to accumulate sublethal damage, two modes of inactivation are identified, namely "ion-kill" (or "intratrack") and " $\gamma$-kill" (or "intertrack"). Cells inactivated by the passage of a single heavy ion are said to be inactivated in the ion-kill mode. The single-particle inactivation cross section for this process is $\sigma$. In the grain-count regime, $\sigma<\sigma_{0}$, and in the track-width regime, $\sigma>\sigma_{0}, \sigma_{0}$ being one of the four cellular radiosensitivity parameters. The fraction of "ion dose" contributing to inactivation by ionkill in the grain-count regime, $P$, is taken to be equal to $\sigma / \sigma_{0}$, while $1-P$ is the fraction of "ion dose" deposited in the $\gamma$-kill mode. For a given ion bombardment, specified by the "effective charge", $Z^{*}$, and relative speed $\beta$ of the ion, $P$ also depends on the radiosensitivity parameters $m$ and $\kappa$. The value of $\kappa$ is so chosen that the transition from the grain- 
count regime to the track-width regime takes place in the neighborhood of $Z^{* 2} / \kappa \beta^{2} \sim 4$ and RBE goes through its maximum when $P \sim 0.5$. Cells not inactivated in the ion-kill mode can be sublethally damaged by the $\delta$ rays from the passing particle and then inactivated, in the $\gamma$-kill mode, by cumulative addition of sublethal damage due to $\delta$ rays from other passing ions. Survival in the $\gamma$-kill mode is taken to follow the $m$-target statistics of inactivation by secondary electrons from X-ray or $\gamma$-ray photons.

In the grain-count regime the surviving fraction of a cellular population whose radiosensitivity parameters are $m, E_{0}, \sigma_{0}$, and $\kappa$, after track-segment irradiation with an ion dose $\mathrm{D}$ of a fluence of $F$ particles of atomic number $Z$, effective charge value $Z^{*}$, relative speed $\beta$, and stopping power $L$ (LET $\infty)$, is a product of survival probabilities in the ionlull mode, $\Pi_{\mathrm{i}}$, and in the $\gamma$-kill mode, $\Pi_{\gamma}$ :

$$
N / N_{0}=\Pi_{\mathrm{i}} \times \Pi_{\gamma} .
$$

The ion-kill mode survival probability is

$$
\Pi_{\mathrm{i}}=\exp (-\sigma F)
$$

where $\sigma$ is the inactivation cross section, $\sigma=\sigma_{0} P$ (see Equation (5)), and $F$ is the ion fluence. The $\gamma$-kill mode survival probability is

$$
\Pi_{\gamma}=1-\left[1-\exp \left[\frac{-D \gamma}{E_{0}}\right]\right]^{m}
$$

where $D_{\gamma}$, the $\gamma$-kill dose fraction is

$$
D_{\gamma}=(1-P) D
$$

and

$$
\frac{\sigma}{\sigma_{0}}=P=\left[1-\exp \left[\frac{-Z^{* 2}}{\kappa \beta^{2}}\right]\right]^{m} .
$$

In the track-width regime, where $P>0.98$, we take

$$
\Pi_{\gamma}=1
$$

and find $\sigma$ from the track width which increases linearly with $Z^{*} / \beta$ while the inactivation cross section increases with $Z^{* 2} / \beta^{2}$ up to a limit set by the maximum radial range of $\delta$ rays. This is the thindown region (6).

To calculate RBE at a given survival level we use the definition

$$
\mathrm{RBE}=D_{\mathrm{x}} / D
$$

where

$$
D_{\mathrm{x}}=-E_{0}\left[\ln \left(1-\left(1-N / N_{0}\right)^{1 / m}\right)\right]
$$

is the X-ray dose after which this level obtains and $D$ is the corresponding ion dose. 
We apply the same formalism to calculate the fraction of transformed cells $K$ in an initial population $N_{0}^{\prime}$ of viable cells (i.e., $K$ represents the fraction of transformants per surviving cell). We write

$$
K=1-N^{\prime} / N_{0}^{\prime}
$$

where $N^{\prime} / N_{0}^{\prime}$ is the fraction of surviving nontransformed cells. The set of transformation radiosensitivity parameters is now $\left(m^{\prime}, E_{0}^{\prime}, \sigma_{0}^{\prime}\right.$, and $\kappa^{\prime}$, and Equation (1) represents the fraction of nontransformed cells, i.e., $1-K$. Equation (2) represents the intratrack (ionkill) probability of nontransformation $\Pi^{\prime}{ }_{i}$, where the inactivation cross section $\sigma$ is now represented by $\sigma^{\prime}$, the "transformation cross section." Similarly, Equation (3) now describes $\Pi_{\gamma}$, the intertrack ( $\gamma$-kill) mode of nontransformation probability, $E_{0}$ and $m$ being replaced by $E_{0}^{\prime}$ and $m^{\prime}$. In Equation (5) $P^{\prime}=\sigma^{\prime} / \sigma_{0}^{\prime}$ is calculated by replacing $\kappa$ and $m$ with $\kappa^{\prime}$ and $m^{\prime}$. The corresponding intertrack dose fraction $D_{\gamma}^{\prime}$ in Equation (4) can then be calculated by replacing $P$ with $P^{\prime}$. In the track-width regime where $P^{\prime}>0.98, \Pi_{\gamma}^{\prime}$ $=1$ and eventually, with decreasing $\beta$, thindown occurs. Radiobiological effectiveness (Equation (7)) and the equivalent X-ray dose $D^{\prime}{ }_{\mathrm{x}}$ (Equation (8)) at a given transformation frequency $K=1-N^{\prime} / N_{0}^{\prime}$ can now be calculated, with $E_{0}^{\prime}$ and $m^{\prime}$ replacing $E_{0}$ and $m$.

All our calculations pertain to water, so the ion dose is always

$$
D=F L .
$$

To calculate the effective charge value of an ion of atomic number $Z$ moving with a relative velocity $\beta$ we use the expression

$$
Z^{*}=Z\left[1-\exp \left(-125 \beta Z^{-2 / 3}\right)\right] \text {. }
$$

We calculate the stopping power in water of an ion of atomic number $Z$ with the aid of Janni's proton stopping power tables (10) and the expression

$$
L(Z, \beta)=L(\mathrm{p}, \beta)\left[Z^{*} / Z_{\mathrm{p}}^{*}\right]^{2},
$$

where $Z^{*}$ and $Z_{\mathrm{p}}^{*}$ are the effective charges of the ion and proton, respectively, (from Equation 11$)$, and $L(\mathrm{p}, \beta)$ is the stopping power, in water, of a proton at the same speed $\beta$.

Ranges of ions in water were also calculated from Janni's tables

$$
R(Z, \beta)=\left(A / Z^{2}\right)\left[R_{\mathrm{p}(\beta)}+\mathrm{C}(\beta)\right]
$$

where $A$ is the ion mass number (in atomic mass unit), $R_{\mathrm{p}(\beta)}$ is the range, in water, of a proton of the same speed $\beta$, and $\mathrm{C}(\beta)$ is a $\beta$-dependent range correction term after Barkas and Berger (11).

Simple programs for calculating track-segment "kill" and survival using Equations (1)-(11) and for calculating stopping power and range for ions in water (Equations (12) (13)) were developed for the mainframe computer and also for the HP-41C hand-held calculator (M. P. R. Waligorski, unpublished).

\section{Results}

In the experiments of Yang et al. (7) the particle beams were filtered through lead foils and detector arrays prior to irradiation of the cell samples, and the reported values of av- 
Table I. Physical Parameters of BEVALAC Ion Beams Used in Track-Segment Calculations

\begin{tabular}{|c|c|c|c|c|c|c|c|c|}
\hline \multicolumn{6}{|c|}{ This work } & \multicolumn{3}{|c|}{ From Yang et al. (7) } \\
\hline \multirow[b]{2}{*}{ El. } & \multicolumn{2}{|c|}{ Radiation } & \multirow{2}{*}{$\begin{array}{c}\text { Beam }^{\mathrm{a}} \\
\text { energy } \\
(\mathrm{MeV} / \mathrm{amu})\end{array}$} & \multirow{2}{*}{$\begin{array}{c}\text { Velocity } \\
\beta\end{array}$} & \multirow{2}{*}{$\begin{array}{c}\text { Track }^{\mathrm{b}} \\
\text { Seg. LET } \\
(\mathrm{MeV} / \mathrm{cm})\end{array}$} & \multirow{2}{*}{$\begin{array}{c}\text { Initial } \\
\text { energy } \\
(\mathrm{MeV} / \mathrm{amu})\end{array}$} & \multirow{2}{*}{$\begin{array}{c}\text { Residual } \\
\text { range }^{b} \\
(\mathrm{~cm})\end{array}$} & \multirow{2}{*}{$\begin{array}{c}\text { Track }^{\mathrm{b}} \\
\text { Avg. LET } \\
(\mathrm{MeV} / \mathrm{cm})\end{array}$} \\
\hline & $Z$ & $A$ & & & & & & \\
\hline $\mathrm{C}$ & 6 & 12 & 438 & 0.733 & 104 & 474 & 31.87 & 100 \\
\hline $\mathrm{Ne}$ & 10 & 20 & 358 & 0.692 & 318 & 425 & 13.88 & 320 \\
\hline $\mathrm{Si}$ & 14 & 28 & 569 & 0.784 & 505 & 670 & 20.58 & 500 \\
\hline $\mathrm{Si}$ & 14 & 28 & 223 & 0.591 & 816 & 320 & 4.5 & 820 \\
\hline $\mathrm{Ar}$ & 18 & 40 & 210 & 0.578 & 1400 & 330 & 3.5 & 1400 \\
\hline $\mathrm{Fe}$ & 2 & 56 & 453 & 0.740 & 1917 & 600 & 8.39 & 1900 \\
\hline $\mathrm{Fe}$ & 26 & 56 & 217 & 0.585 & 2860 & 400 & 2.5 & 3000 \\
\hline $\mathrm{Fe}$ & 26 & 56 & 103 & 0.435 & 4751 & 300 & 0.7 & 5000 \\
\hline $\mathrm{U}$ & 92 & 238 & 943 & 0.868 & 18600 & 960 & 8.6 & 19000 \\
\hline
\end{tabular}

${ }^{a}$ At the point of irradiation.

${ }^{\mathrm{b}}$ In water.

erage LET were estimated on the basis of the measured residual ranges for each beam. Using these range values, we have calculated the track-segment energy, $\beta$, and LET values at the position of the irradiated cells for each beam. Results of these calculations are listed in Table I.

From the measured survival data after X irradiation, we have extracted $m=3$ and $E_{0}$ $=1.7$ Gy for cells plated immediately after irradiation (instantaneous plating) and $m=3$ and $E_{0}=2.8 \mathrm{~Gy}$ for cells plated $24 \mathrm{~h}$ after irradiation (delayed plating). Assignment of $\kappa=750$ and $\sigma_{0}=5 \times 10^{-7} \mathrm{~cm}^{2}$ for both cases allowed us to reproduce the measured survival data, as shown in Figure 1, and to calculate survival RBE-LET dependences, according to Equations (1)-(13), by varying the speed of ion species listed in Table I. An example of a comparison between the calculated and measured RBE values at $10 \%$ surviving fraction for cells plated immediately after irradiation is shown in Figure 2. The overall agreement between the calculated and reported values of RBE is about $15 \%$ or better.

Assignment of radiosensitivity parameters for radiation-induced transformations is much more difficult. The X-ray data, shown in Figure 3, permitted us to use $m=2$ or $m$ $=3$ target models and fit corresponding values of $E_{0}$. Over a range of possible assignments, we chose the set $\left(m, \kappa, \sigma_{0}, E_{0 \text {,inst }}\right.$ and $\left.E_{0, \text { del }}\right)=\left(2,750,1.15 \times 10^{-10} \mathrm{~cm}^{2}, 180 \mathrm{~Gy}\right.$, and $260 \mathrm{~Gy}$ ) to reproduce the experimental data, as shown in Figure 4, and to calculate the RBE-LET dependences shown in Figure 5.

Following Yang et al., RBE values were calculated for transformation frequencies after doses of $\mathrm{X}$ rays giving $50 \%$ survival. This corresponded to a calculated dose of $2.68 \mathrm{~Gy}$ (transformation frequency $2.18 \times 10^{-4}$ ) for instantaneous plating and to $4.42 \mathrm{~Gy}$ $\left(2.8 \times 10^{-4}\right)$ for delayed plating in the case of the $m=2$ target parameters.

The $m=3$ target parameter set $\left(3,475,0.7 \times 10^{-10} \mathrm{~cm}^{2}, 50 \mathrm{~Gy}\right.$, and $\left.75 \mathrm{~Gy}\right)$ also reproduced experimental data to a similar degree. An example of RBE-LET calculations using this set of parameters is shown in Figure 6. Here, the corresponding calculated 


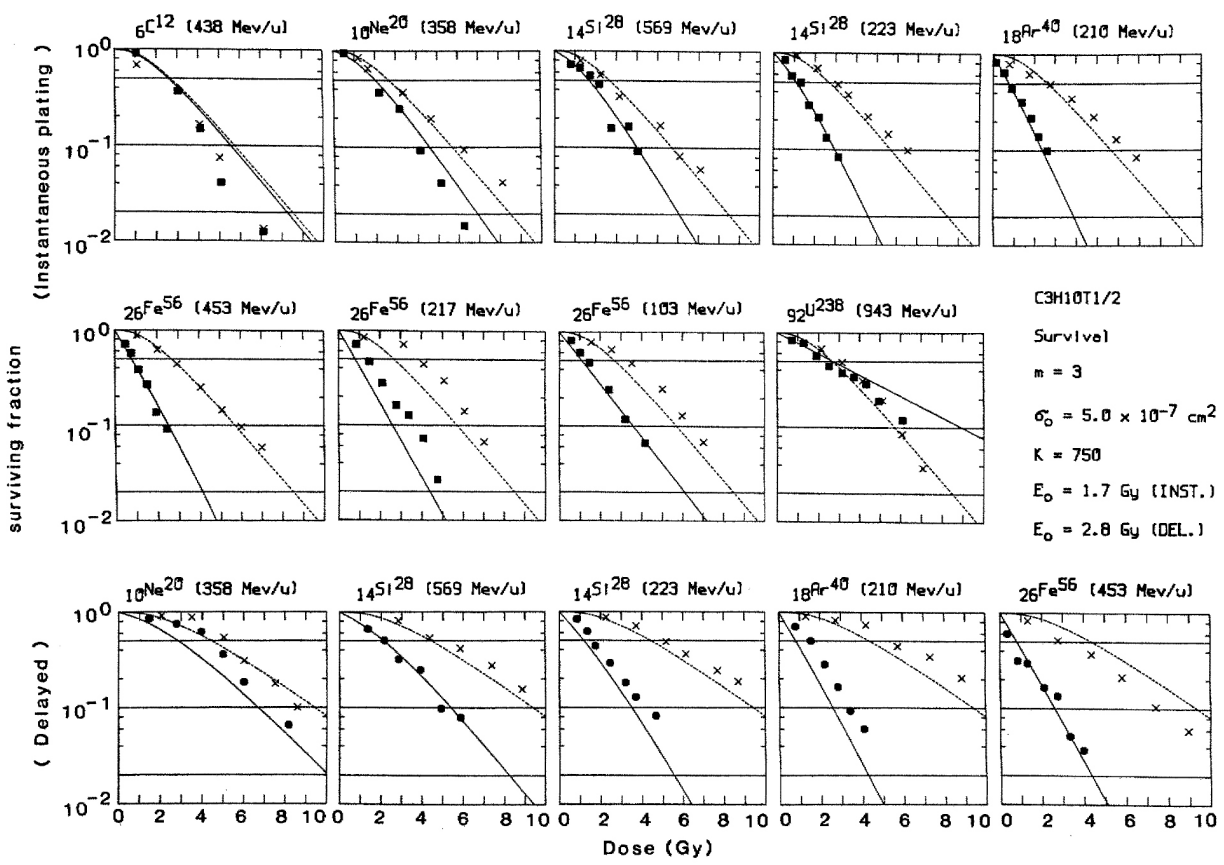

Figure 1. Measured (7) and calculated survival fraction of C3H10T1/2 cells after irradiation with $X$ rays $(\mathrm{X})$ and BEVALAC ions, for instantaneous (squares) and delayed (circles) plating. Curves for $\mathrm{X}$ rays (broken lines) and for heavy ion bombardments indicated above each panel were all calculated using the four radiosensitivity parameters listed, with different values of $E_{0}$ for instantaneous and delayed plating. Survival levels of 50,10, and $2 \%$ are drawn as horizontal lines in each panel.

transformation frequencies at 2.68 and 4.42 Gy were $1.42 \times 10^{-4}$ and $1.87 \times 10^{-4}$ for instantaneous and delayed plating, respectively.

Using these two sets of parameters we were able to reproduce the reported values of $\mathrm{RBE}$ for transformations within $25 \%$ or better for all heavy ion beams except the ${ }_{18}^{40} \mathrm{Ar}$ beam.

\section{Discussion}

To extract radiosensitivity parameters for track theory calculations we need experimental data measured under conditions of track-segment irradiation by heavy ions of widely ranging charge numbers and energies. The systematic experimental survey of Yang et al. allowed us, for the first time, to extract model parameters for radiation-induced neoplastic transformations in the $\mathrm{C} 3 \mathrm{H} 10 \mathrm{~T} 1 / 2$ system. Results of experiments where this system had been exposed to other high-LET radiations, such as fission neutrons (12) and both fission and cyclotron-produced neutrons (13), do not lend themselves to parameterization since they are the outcome of a complicated averaging process in which many short-range secondary practices participate, as we see in model calculations (14). 


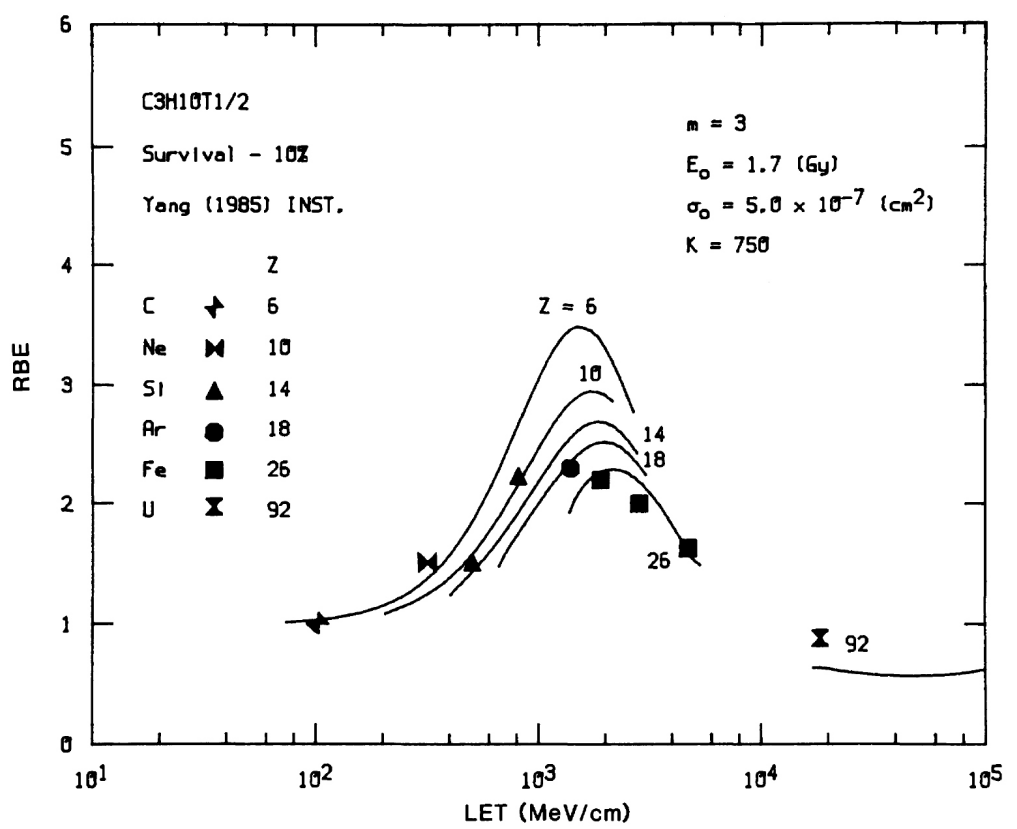

Figure 2. Measured (7) and calculated RBE at 10\% survival for cells plated immediately after irradiation.

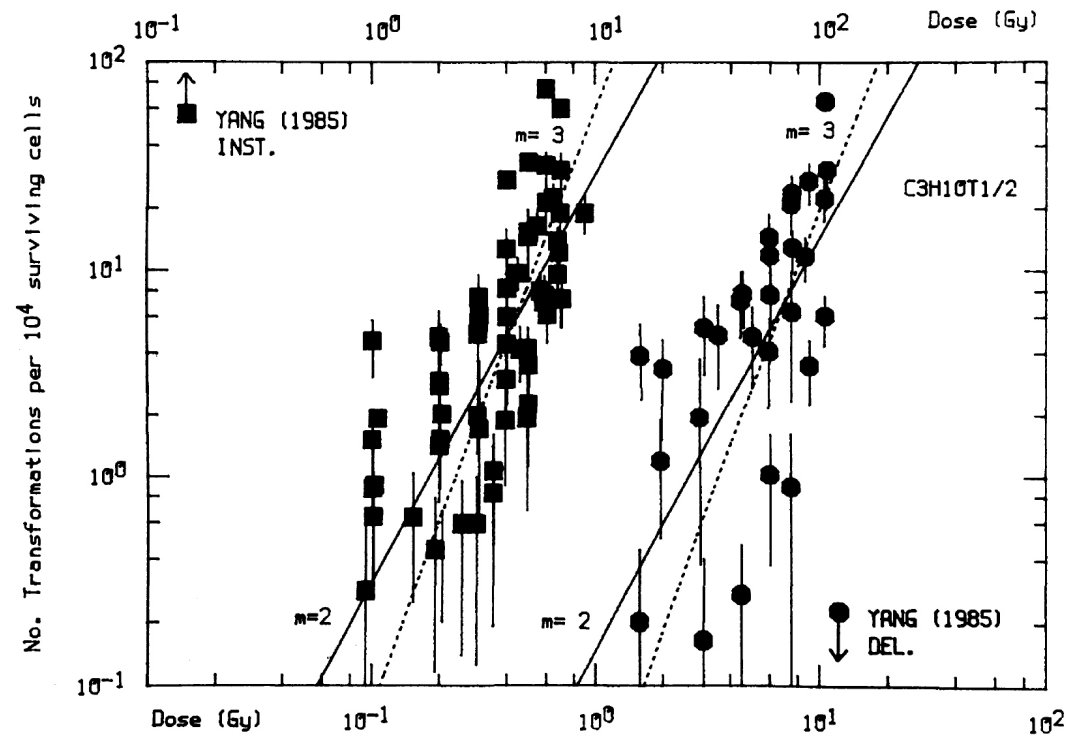

Figure 3. Measured (7) transformation frequencies after X-ray doses for instantaneous (INST.upper abscissa) and delayed (DEL.- - lower abscissa) plating. Full line represents the $m=2$ target model $\left(E_{0, \text { inst }}=180 \mathrm{~Gy}, E_{0 \text {,del }}=260 \mathrm{~Gy}\right)$. Broken line represents $m=3\left(E_{0, \text { inst }}=50 \mathrm{~Gy}, E_{0, \text { del }}=75\right.$ Gy). 


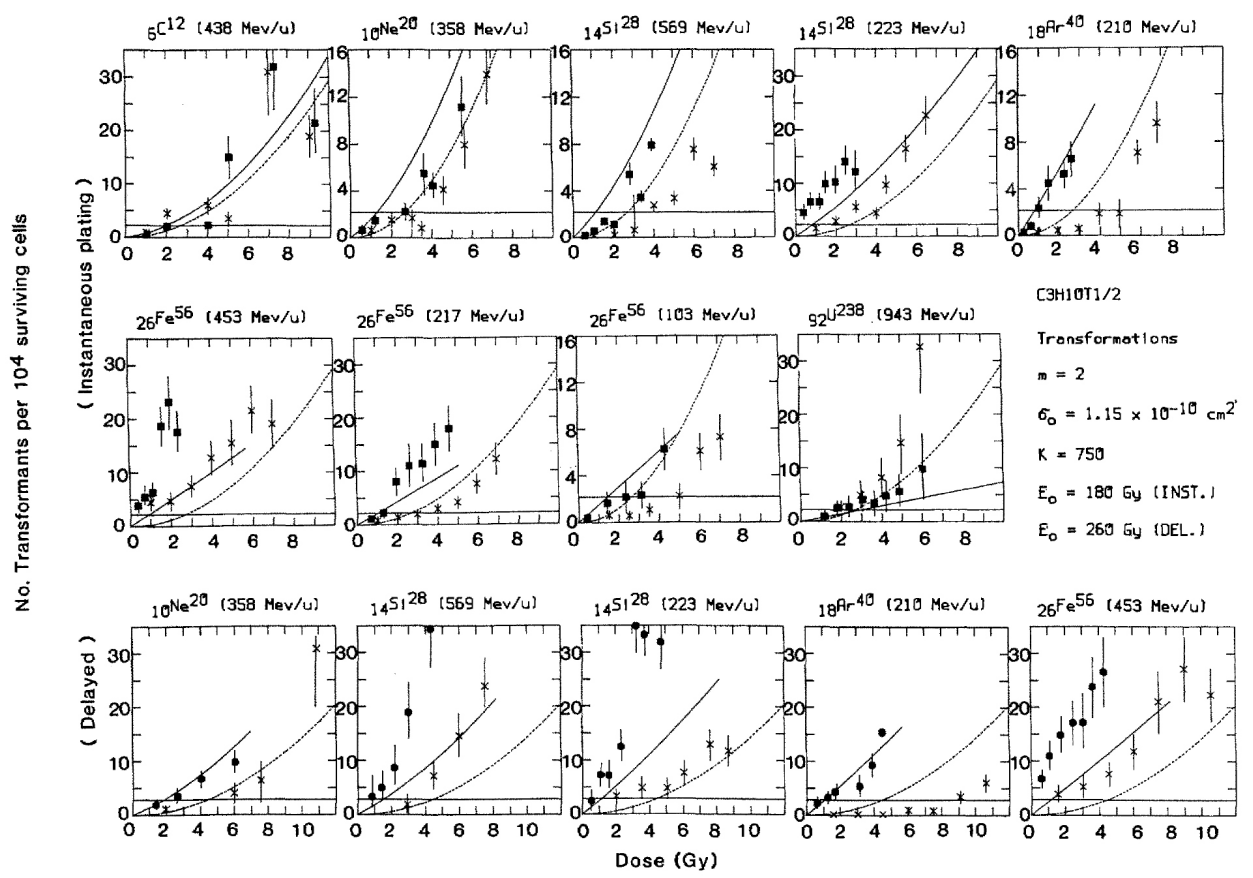

Figure 4. Measured (7) and calculated transformation frequencies in $\mathrm{C} 3 \mathrm{H} 10 \mathrm{~T} 1 / 2$ cells after irradiation with $\mathrm{X}$ rays $(\mathrm{X})$ and BEVALAC ions for instantaneous (squares) and delayed (circles) plating. Curves for X rays (broken lines) and for heavy ion bombardments indicated above each panel (solid lines) were calculated using the $m=2$ target model parameters shown, with different values of $E_{0}$ for instantaneous and delayed plating. Transformation frequencies corresponding to $50 \% \mathrm{X}$ ray survival are drawn as horizontal lines in each panel.

In another study of survival (15) and transformations (16) after $\alpha$-particle irradiation by Lloyd and co-workers, the X-ray data have not been published, making an evaluation of $m$ and $E_{0}$ uncertain.

We note the wide range of reported values of the extrapolation number in $X$-ray survival curves measured for this system: from 1.8 (for $100 \mathrm{kVp} \mathrm{X}$ rays) (17) to 5.6 (for 50 $\mathrm{kVp} \mathrm{X}$ rays) (12) to 5.4 (again, for $100 \mathrm{kVp} \mathrm{X}$ rays) (13). Judging from the fair reproduction of Yang's survival data (measured after $225 \mathrm{kVp}$ X rays) presented in Figure 1, we are reasonably confident of our value $m=3$. Our cellular parameters for C3H10T1/2 survival closely resemble those found for aerated Chinese hamster or HeLa cells (18). In the other mammalian cells examined in this study, values of $m$ exceeding 3 have not been found.

It is interesting that two groups of investigators report similar extrapolation numbers of 4.5 (12) and 5.4 (13) for C3H10T1/2 survival after irradiation with fission neutrons, from which we would infer a similar $\gamma$-ray contamination of their neutron beams. This apparently is not so, since $\gamma$-ray contributions to the total dose of $4 \%$ (12) and $8-20 \%$ (13) are specified. The effect of both those neutron beams on cellular survival arises mainly from slow protons in the ion-kill mode (3); hence different shoulders in the measured survival curves should reflect the differences in the $\gamma$-ray contamination of the neu- 


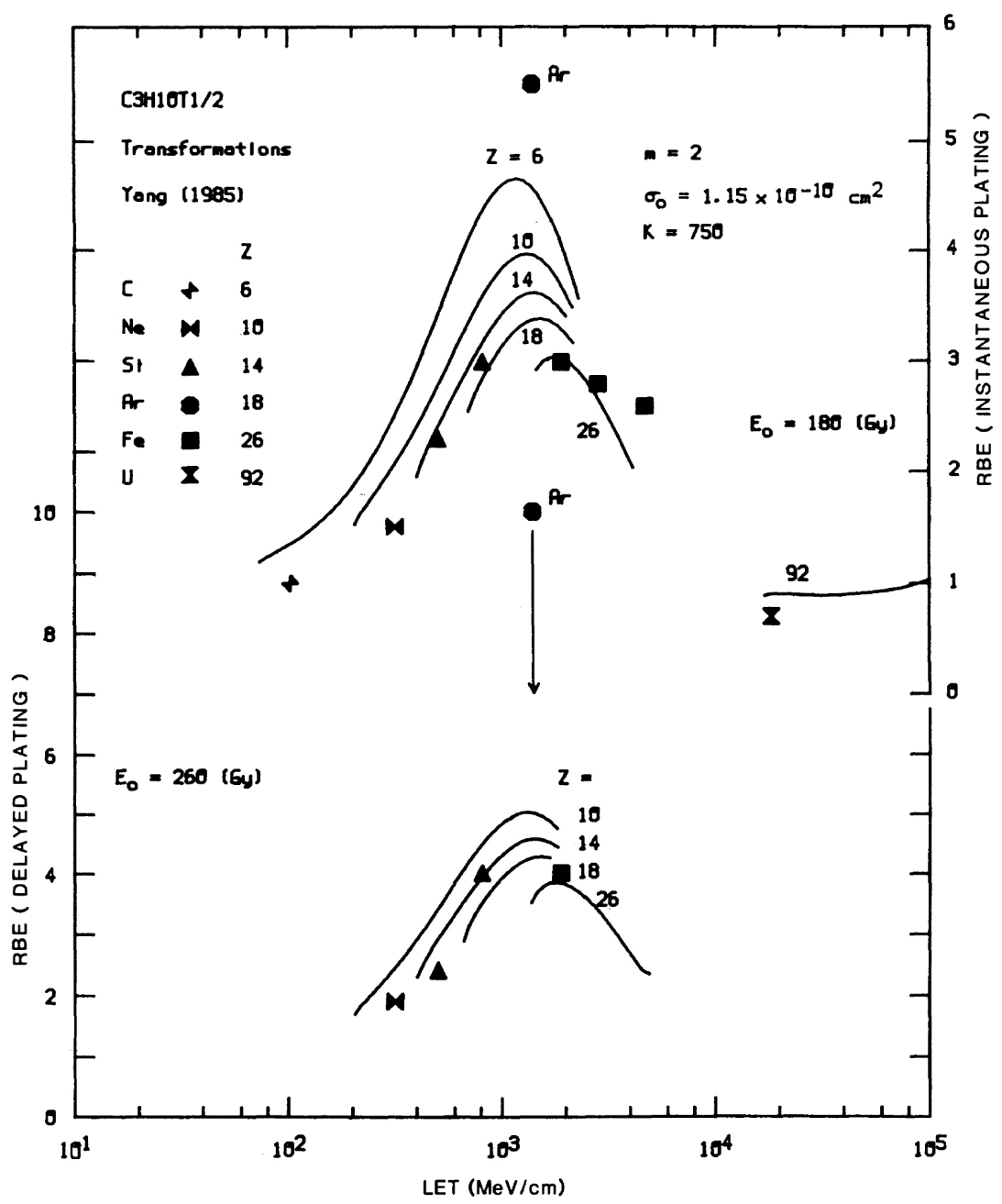

Figure 5. Measured (7) and calculated RBE for transformations in C3H10T1/2 cells at levels corresponding to $50 \%$ survival, indicated in Figure 4, for instantaneous (upper panel, upper righthand ordinate) and delayed (lower panel, lower left-hand ordinate) plating. $m=2$ target parameters were used.

tron beams. No initial shoulder has been reported in the purely exponential C3H10T1/2 survival curves measured after irradiation with $5.6 \mathrm{MeV} \alpha$ rays $(15,16)$ where no $\gamma$-ray contamination is present and the ion-kill mode also predominates.

Our estimates of radiosensitivity parameters for radiation-induced transformations in the $\mathrm{C} 3 \mathrm{H} 10 \mathrm{~T} 1 / 2$ cell line are much more tenuous; however, both sets of parameters, for the $m=2$ and $m=3$ target models, seem to reproduce the measured values of RBE quite well (see Figure 5 and Figure 6). On comparison with Figure 4, one may agree with Yang et al. that their experimental procedures could have introduced different dosemodifying for experiments performed with different beams. In view of these factors and 


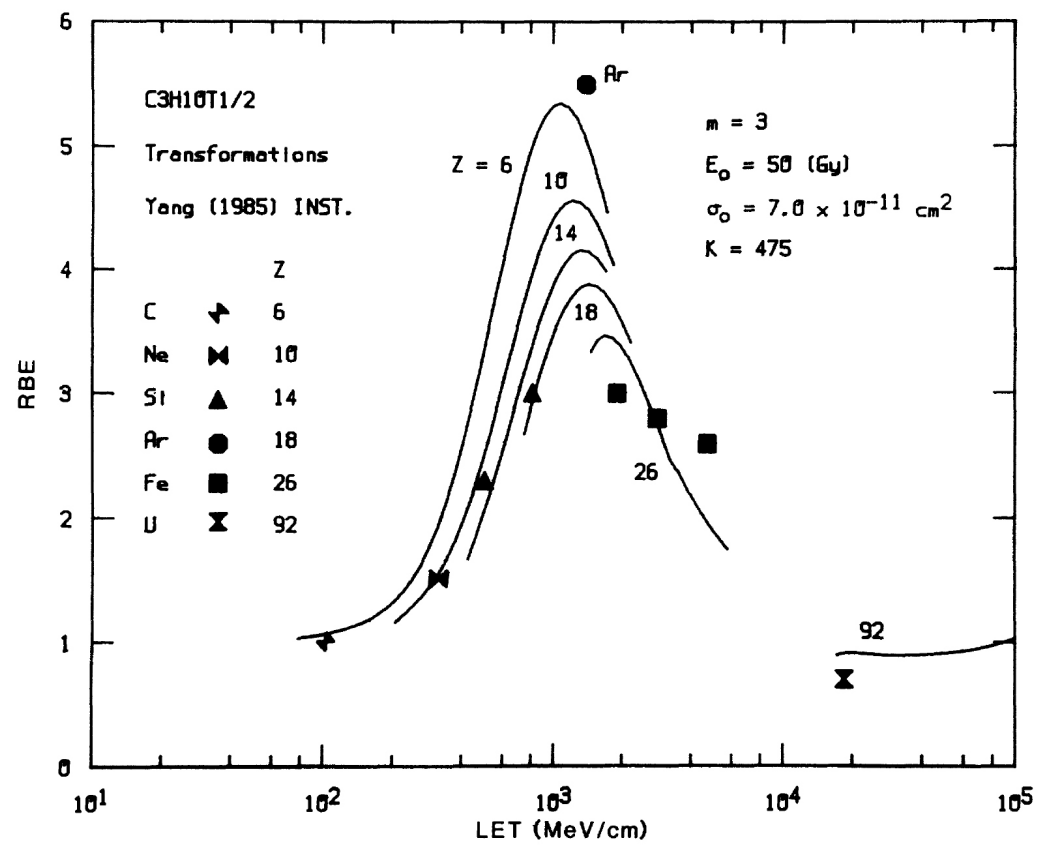

Figure 6. Measured (7) and calculated RBE for transformations in C3H10T1/2 cells for instantaneous plating. $m=3$ target parameters were used in this calculation.

due to large experimental errors in Yang's data, the application of routine tests to assess the quality of fits seems inappropriate.

Most investigators find a quadratic dose dependence for X-ray-induced transformations in the C3H10T1/2 system $(12,13,17,19)$ above 1 Gy and below 4 Gy, while a more complicated dependence is indicated below $1 \mathrm{~Gy}$ and saturation is observed above X-ray doses exceeding 4 Gy.

We note that in our calculations we treat cellular lethality and transformation induction as statistically independent processes. The radiosensitivity parameters $E_{0}$ and $\sigma_{0}$ for the two processes differ by orders of magnitude: for transformation $E_{0}=180 \mathrm{~Gy}$, while for cell killing $E_{0}=1.7 \mathrm{~Gy}$; for transformation $\sigma=1.5 \times 10^{-10} \mathrm{~cm}^{2}$ and for killing $\sigma_{0}=$ $5.7 \times 10^{-7} \mathrm{~cm}^{2}$, supporting this conjecture. Another view of these processes is offered by Goodhead (20). We take it that the surviving cells after irradiation represent a random sample of the irradiated cells in which the experimental assay of transformed cells has been made by measuring the number of transformed and surviving cells. It is our approximation that the same fraction of transformed cells would be found in any sample of the original population, but that the assay cannot be made for cells which cannot form colonies.

Our calculations of RBE clearly demonstrate the nonunique dependence of RBE on LET, both for survival and transformations (Figure 2, Figure 5, and Figure 6). As expected, $(1-3,21)$ the particle of lowest charge gives the highest calculated maximum value of RBE, the maxima in the RBE curves shifting toward higher values of LET with increasing charge of the bombardment. Another parameterization of RBE, through 


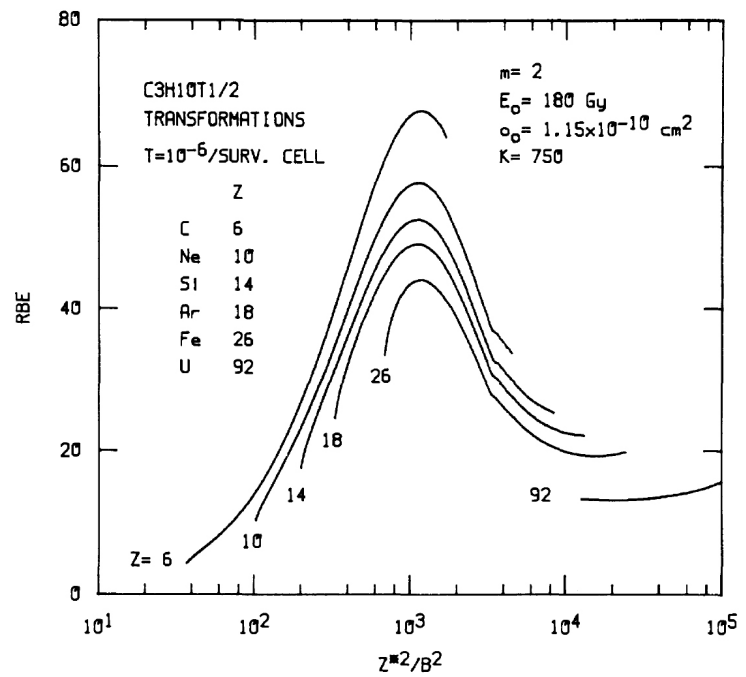

Figure 7. RBE vs $Z^{* 2} / \beta^{2}$ calculated for BEVALAC ions at a transformation frequency per surviving cell. $m=2$ target parameters (instantaneous plating) were used in these calculations. This transformation frequency corresponds to an X-ray dose of $18 \mathrm{cGy}$ and 0.990 survival. Note the vertical displacement of RBE curves with the charge $Z$ of the bombardment.

$Z * 2 / \beta^{2}$, does not alleviate the problem, however, reducing the "LET-shift" of the RBE curves (4), as demonstrated in Figure 7 where we predict RBE for transformations (instantaneous plating) at the lowest frequency of spontaneous cell transformations observed by Yang, i.e., $10^{-6}$ transformants per surviving cell. According to our calculations, this corresponds to an X-ray dose of $18 \mathrm{cGy}$, which translates to a survival fraction of 0.990 , as calculated for the X-ray survival curve of Figure 1 (instantaneous plating).

We believe that a wider choice of ion species and of their energy would help to demonstrate better the nonuniqueness of the RBE-LET dependence for survival and neoplastic transformations in the $\mathrm{C} 3 \mathrm{H} 10 \mathrm{~T} 1 / 2$ system, especially around the thin-down region, in a manner already demonstrated for survival of Chinese hamster cells (5).

Concern has been expressed (22) about absurdly high RBEs in the low-dose limit which might result from describing the response to $\gamma$ rays by a multitarget model. The values of RBE shown in Figure 5, Figure 6, and Figure 7 correspond to X-ray doses of $2.68,2.68$, and $18 \mathrm{cGy}$, respectively, and do not appear to be excessively high. Fry et al. (8) report an experimental value of $\mathrm{RBE}=27$ for in vivo tumorigenic effect in the Harderian gland of pituitary-isografted mice exposed to $600 \mathrm{MeV} / \mathrm{u} \mathrm{Fe}$ ions in the plateau region over a dose range $0-40 \mathrm{cGy}$. Our calculations of transformation RBE, performed for Fe ions of energy $453 \mathrm{MeV} / \mathrm{u}$, at a transformation level of $2.5 \times 10^{-9}$ per survivor (corresponding to a dose of $10 \mu \mathrm{Gy}=1 \mathrm{mrad}$, or a fluence of 33 ions $/ \mathrm{cm}^{2}$ ) yield values of ca 900 and 13,000 for the $m=2$ and $m=3$ parameter sets, respectively. While these values may seem high, they are perhaps not unreasonable.

At these extremely low fluences, dose-based concepts like RBE tend to lose their significance. 
In view of some experimental evidence that a truly flat shoulder may exist in the survival curve for CHO cells (23), the issue of extrapolating RBE to extremely low doses may have to be reviewed more carefully.

We cannot explain the difference between the observed and calculated values of transformation RBE for the $210 \mathrm{MeV} / \mathrm{amu}$ Ar beam (see Figure 5), even though the respective values for survival RBE appear to be reasonably consistent (Figure 1 and Figure 2). Perhaps new measurements of transformations for this irradiation will show better consistency with the remaining experimental data and better agreement with our calculations.

We account for the apparently increased radioresistance of cells plated $24 \mathrm{~h}$ after irradiation (delayed plating) by modifying the value of $E_{0}$ in a manner employed earlier for predicting survival under aerobic and anoxic conditions (18).

From our analysis of transformation after X-ray and high-LET irradiation of the C3H10T1/2 cell system, we conclude that the extrapolation to low X-ray doses is most likely quadratic or perhaps cubic, and that our extracted radiosensitivity parameters allow us to reasonably predict transformation frequencies in this system for an arbitrary radiation environment, at least in the presence of "average" dose-modifying factors.

It is tempting to extend our findings to in vivo systems, where pure-quadratic lowdose extrapolations of X-ray-induced cancers have also been made for myeloid leukemia in male CBA/H mice (24) and in rat skin carcinogenesis (25). Let us assume proportionality between the probability of cell transformation in an organ and the probability of finding a tumor growth in it, and also that our values of transformation radiosensitivity parameters found for C3H10T1/2 cells are applicable to other cells in vivo. We then ask how many transformations at the cellular level are needed to "generate" observable radiation-induced cancer in vivo.

For illustration, we give an example of such a calculation: Fry et al. $(8,26)$ have studied the incidence of tumors induced in the Harderian gland of pituitary-isografted mice and found for a dose of $5 \mathrm{cGy}$ of $453 \mathrm{MeV}$ Fe ions in the ionization plateau region a cancer incidence of ca. $14 \%$. Let us assume the mammalian cell dimensions to be $10 \times 10 \times 10 \mu^{3}$ (i.e., that the number of cells in $1 \mathrm{~cm}^{3}$ is $N=10^{9}$ ) and the volume of the Harderian gland to be $1 \mathrm{~mm}^{3}$. The number of gland cells in which transformations occur is $T=N \sigma F$, where $F$, the particle fluence, is ca. $1.6 \times 10^{5} \mathrm{ions} / \mathrm{cm}^{2}$, and $\sigma$ is the cross section, equal to $\sigma_{0} P$ (see Equation (6)). Let us take $\sigma_{0}=1.15 \times 10^{-10} \mathrm{~cm}^{2}$, from the $m=$ 2 target set of transformation parameters. From Equation [6], we calculate $P=0.82 . T$ is then equal to about 20,000 transformations $/ \mathrm{cm}^{3}$, or about 20 cells would become transformed in the Harderian gland under these irradiation conditions. From the observed cancer incidence of $14 \%$ (8) we estimate that it takes about 150 transformed cells to initiate an observable tumor growth in this organ.

This example does not claim to represent the mechanisms involved in radiation-induced carcinogenesis. We discuss it to illustrate the manner in which track structure calculations could be used as a basis for estimating the in vivo radiation hazard by exploiting the predictive capability of the model at the cellular level. Even in its present four-parameter formulation, track theory offers a testable hypothesis on which assessments of biological hazards of radiation could be based and more realistic limits for purposes of radiation protection derived (27). 


\section{Conclusions}

Track structure theory offers a four-parameter reduction of experimental measurements of neoplastic transformations induced in C3H10T1/2 cells by energetic heavy ions in a manner similar to that found earlier for cellular survival. This theory provides us with a consistent scheme for extrapolation to low doses of low-LET radiation from high doses of high-LET radiations. While the experimental results of Yang et al. do not permit us to make more definite parameter assignments, we are able to state that when extrapolated to the low-dose region, the transformation frequency in these cells depends on the square (or perhaps even cube) of the X-ray dose. This could bear on the presently established limits for low-dose radiation hazard. Clearly, a better understanding and more experimental efforts are necessary. Since the track $\delta$-ray approach offers a reasonably clear distinction between the physical and biological aspects of the problems involved, it should serve as one of the theoretical guidelines in these investigations.

\section{Acknowledgments}

We thank Givargis Danialy and Kim Sun Loh for their help in performing calculations and preparing computer graphics. One of the authors (M.P.R.W.) acknowledges the support of the International Atomic Energy Agency (Vienna) in the form of a fellowship. This work has been supported by the U.S. Department of Energy.

\section{References}

1. R. Katz, S. C. Sharma, and M. Homayoonfar, The structure of particle tracks. In: Topics in Radiation Dosimetry (F. H. Attix, Ed.). Academic Press, New York, 1972.

2. R. Katz, Track structure theory in radiobiology and in radiation detection. Nucl. Track Detect. 2, 1-28 (1978).

3. R. Katz and S. C. Sharma, Response of cells to fast neutrons, stopped pions, and heavy ion beams. Nucl. Instrum. Methods 110, 93-116 (1973).

4. J. Thacker, A. Stretch, and M. A. Stephens, Mutation and inactivation of cultured mammalian cells exposed to beams of accelerated heavy ions II. Chinese Hamster V79 cells. Int. J. Radiat. Biol. 36, 137-148 (1979).

5. H. Wulf, W. Kraft-Weyrather, H. G. Miltenburger, E. A. Blakely, C. A. Tobias, and G. Kraft, Heavy ion effects on mammalian cells: Inactivation measurements with different cell lines. Radiat. Res. 104, Suppl. 8, S122-S134 (1985).

6. R. Katz, D. E. Dunn, and G. L. Sinclair, Thindown in radiobiology. Radiat. Prot. Dosim. 13, 281-284 (1985).

7. T. C. Yang, L. M. Craise, and C. A. Tobias, Neoplastic cell transformation by heavy charged particles. Radiat. Res. 104, Suppl. 8, S177-S187 (1985).

8. R. J. M. Fry, P. Powers-Risius, E. L. Alpen, and E. J. Ainsworth, High-LET radiation carcinogenesis. Radiat. Res. 104, Suppl. 8, S188-S195 (1985).

9. R. Katz and E. J. Kobetich, Particle tracks in emulsion. Phys. Rev. 186, 344-351 (1969).

10. J. F. Janni, Proton range-energy tables, $1 \mathrm{KeV}-10 \mathrm{GeV}$. At. Data Nucl. Data Tables 27, 147529 (1982). 
11. W. H. Barkas and M. J. Berger, Tables of energy losses and ranges of heavy charged particles. In Studies in Penetration of Charged Particles in Matter, pp. 103-172. National Academy of Science, National Research Council, Washington, DC, 1964.

12. A. Han and M. M. Elkind, Transformation of mouse C3H/10T1/2 cells by single and fractionated doses of X-rays and fission spectrum neutrons. Cancer Res. 39, 123-130 (1979).

13. E. K. Balcer-Kubiczekand G. H. Harrison, Oncogenic transformation of C3H/10T1/2 cells by X-rays, fast-fission neutrons, and cyclotron-produced neutrons. Int. J. Radiat. Biol. 44, 377 (1983).

14. R. Katz and S. C. Sharma, Cellular survival in a mixed radiation environment. Int. J. Radiat. Biol. 26, 143-146 (1974).

15. E. L. Lloyd, M. A. Gemmell, C. B. Henning, D. S. Gemmell, and B. J. Zabransky, Cell survival following multiple track alpha particle irradiation. Int. J. Radiat. Biol. 35, $23-31$ (1979).

16. E. L. Lloyd, M. A. Gemmell, C. B. Henning, D. S. Gemmell, and B. J. Zabransky, Transformation of mammalian cells by alpha particles. Int. J. Radiat. Biol. 36, 467-478 (1979).

17. M. Terzaghi and J. B. Little, X-radiation-induced transformation in a $\mathrm{C} 3 \mathrm{H}$ mouse embryo-derived cell line. Cancer Res. 36, 1367-1374 (1976).

18. R. A. Roth, S. C. Sharma, and R. Katz, Systematic evaluation of cellular radiosensitivity parameters. Phys. Med. Biol. 21, 491-503 (1976).

19. C. Borek and E. J. Hall, Induction and modulation of radiogenic transformation in mammalian cells. In Radiation Carcinogenesis: Epidemiology and Biological Significance (J. D. Boice, Jr., and J. F. Fraumeni, Jr., Eds.), pp. 291-302. Raven Press, New York, 1984.

20. D. T. Goodhead, Deductions from cellular studies of inactivation, mutagenesis, and transformation. In Radiation Carcinogenesis: Epidemiology and Biological Significance (J. D. Boice, Jr., and J. F. Fraumeni, Jr., Eds.), pp. 369-385. Raven Press, New York, 1984.

21. R. Katz, Two calculations from track theory. In Seventh Symposium on Microdosimetry (J. Booz, H. G. Ebert, and H. D. Hartfiel, Eds.), pp. 583-590. Harwood, London, 1980.

22. D. T. Goodhead, Quality factor for low doses of high-LET radiations: Comments on the proposal of Hofmann and Katz. Health Phys. 46, 237-239 (1984).

23. R. L. Warters, K. G. Hofer, C. R. Harris, and J. M. Smith, Radionuclide toxicity in cultured mammalian cells: Elucidation of the primary site of radiation damage. Curr. Top. Radiat. Res. Q. 12, 389-407 (1977).

24. R. H. Mole, Dose-response relationships. In Radiation Carcinogenesis: Epidemiology and Biological Significance (J. D. Boice, Jr., and J. F. Fraumeni, Jr., Eds.), pp. 403-420. Raven Press, New York, 1984.

25. F. J. Burns and R. E. Albert, Application of a linear quadratic model with repair to rat skin carcinogenesis data. In Proceedings, 7th International Congress of Radiation Research (J. J. Broerse, G. W. Barendsen, H. B. Kal, and A. J. Van der Kogel, Eds.), p. C6-05. Martinus Nijhoff, Amsterdam 1983.

26. R. J. M. Fry, P. Powers-Risius, E. L. Alpen, E. J. Ainsworth, and R. L. Ullrich, High-LET radiation carcinogenesis. Adv. Space Res. 3, 241-248 (1983).

27. W. Hofmann and R. Katz, Track structure analysis of radiation carcinogenesis at low doses. In 8th Symposium in Microdosimetry (J. Booz and H. G. Ebert, Eds.), pp. 565-573. Commission of the European Communities, Brussels, 1982. 